\title{
Spatial Distribution of Construction Waste Recycling Facilities
}

\author{
Si-Yan Gao, ${ }^{1 *}$ Yang Liu, ${ }^{1,2,3^{* *}}$ Yuan-Yang Zhou, ${ }^{1}$ and Min Zhang ${ }^{1}$ \\ ${ }^{1}$ School of Geomatics and Urban Spatial Informatics, Beijing University of Civil Engineering and Architecture, \\ No.15 Yongyuan Rd., Daxing District, Beijing 102616, China \\ ${ }^{2}$ Beijing Advanced Innovation Center for Future Urban Design, Beijing University of Civil Engineering and \\ Architecture, No.1 Zhanlanguan Rd., Xicheng District, Beijing 100044, China \\ ${ }^{3}$ Beijing Key Laboratory of Urban Spatial Information Engineering, \\ No.15 Yangfangdian Rd., Haidian District, Beijing 100038, China
}

(Received August 18, 2020; accepted October 27, 2020)

Keywords: construction waste, resource facilities, GIS analysis, spatial pattern, Beijing

With the acceleration of urban construction and transformation, the problems of construction waste pollution and garbage are becoming increasingly serious. This study focuses on the distribution characteristics and relationship of 369 construction waste storage sites and 115 construction waste recycling facilities sites in Beijing municipal district, using a geographic information system (GIS) spatial analysis method as a way to counter the problems of subjectivity and the lack of a scientific basis in determining how construction waste recycling should be planned. The results show that (1) the amount of construction waste generated in Beijing indicates the characteristics of circle differentiation from the core to the edge, and the southern part of the city, which is $10-30 \mathrm{~km}$ away from the city center, is where the concentration is highest; (2) the distribution of construction waste recycling facilities in Beijing is uneven, with the greatest concentration located outside the Fifth Ring Road on the periphery of the city; (3) the area with the largest capacity of construction waste recycling facilities is within the standard deviation ellipse (SDE) range of 700000 tons/year with the coordinates $(116.4437,39.9655)$ as the center, a long axis of $43.6 \mathrm{~km}$, and a short axis of $23.8 \mathrm{~km}$; (4) the effective coverage area of Beijing construction waste recycling facilities is $72.6 \%$, which does not completely cover all construction waste storage sites. The results of this study serve as reference for the treatment of construction waste and the location of resource facilities in Beijing.

\section{Introduction}

With the continuous expansion of cities in China, the disparity between the increase in construction waste output and the lag in the construction of treatment facilities has become increasingly prominent. ${ }^{(1)}$ By 2020, the output of construction waste in China will reach 2.6 billion tons, accounting for $30-40 \%$ of the total municipal solid waste generated. Much construction waste is dumped in landfills or at unmonitored sites, ${ }^{(2)}$ which occupy a large

\footnotetext{
*Corresponding author: e-mail: gsy15810324480@163.com

** Corresponding author: e-mail: liuyang@bucea.edu.cn

https://doi.org/10.18494/SAM.2020.3061
} 
area of land, create potential safety hazards caused by excessive stacking, and cause air and groundwater pollution. Many cities are faced with the problems of land shortage and storage yards of construction waste that cannot process the amount of waste generated.

With increasing calls for a circular economy around the world, the appropriate management of demolition waste has emerged as a key challenge. ${ }^{(3)}$ Utilizing construction waste as a resource for the emerging environmental protection industry is the most ideal method for treating construction waste. To achieve the aims of green and low-carbon, resource regeneration, comprehensive utilization, and serving the society, it is urgent that modern construction projects treat and reuse construction waste and residual mud and debris and promote the development of resource utilization, waste reduction, and high efficiency. However, the utilization of construction waste as a resource is still in the initial stage in China; the rate of utilization is currently below $10 \%$. The insufficient number and inefficient distribution of resource facilities are also important factors contributing to this situation; in addition, the resource processing technology needs to be improved.

Geographic information system (GIS) spatial analysis methods are effective for studying the spatial layout of facilities. Weiting and Yungian ${ }^{(4)}$ used standard deviation ellipse (SDE), kernel density estimation, and neighborhood and SPSS correlation analyses to study the spatial distribution characteristics and influencing factors of public cultural facilities in Xi'an. Xiaofeng et al. ${ }^{(5)}$ applied a GIS spatial analysis method to establish a systematic technique to extract the spatial distribution characteristics of urban transportation facilities in Kunming from 2016 to 2018 based on the directional distribution, spatial agglomeration, and hot spot estimation for point of information (POI) data. Buber et al. ${ }^{(6)}$ compiled a spatial distribution map based on GIS point density analysis and determined the areas at risk of air pollution caused by domestic shipping in Izmir Bay using a bottom-up emission assessment method. Burns et al. ${ }^{(7)}$ used the Poisson regression method to evaluate the spatial grouping of infants with low birth weight and the geographical distribution of maternal risk factors to determine the spatial relationship between risk factors and low birth weight. Many previous studies focused on selecting ideal landfill sites for municipal solid waste and disposal sites for resource facilities using GIS spatial methods and multistandard comprehensive decision making methods; ${ }^{(8)}$ however, further research is needed on the relationship between the spatial distribution and location of built resource facilities.

Therefore, to promote the coordination between urban development and the urban population, resource use, and the environment, and increase waste reduction and recycling, in this study, we applied the average nearest-neighbor index, kernel density function, SDE, and buffer analyses to data on resource facilities and construction waste dump sites in Beijing. We explored the characteristics of the spatial layout and agglomeration of construction waste and related recycling facilities in Beijing in a manner that can inform urban planning and the selection of urban construction waste recycling facility sites in Beijing. 


\section{Materials and Methods}

\subsection{Study area and data source}

We consider Beijing as the study area. Beijing has a total area of $16400 \mathrm{~km}^{2}$ and 16 districts under its jurisdiction. According to the Beijing Urban Master Plan, Beijing is divided into capital functional core areas (Dong Cheng and Xicheng Districts), urban functional expansion areas (Hai Dian, Chaoyang, Shi Jingshan, and Feng Tai Districts), new urban development areas (Chang Ping, Shun Yi, Tong Zhou, Da Xing, and Fang Shan Districts), and ecological

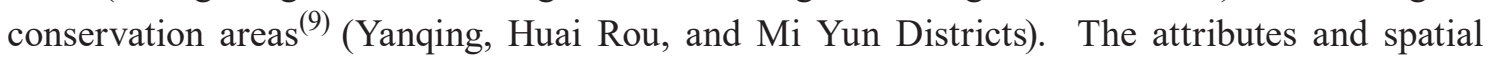
data on the resource facilities are from the Beijing Construction Waste Management Platform and AutoNavi's POI data, respectively. Monthly data on resource facilities are exported from the construction waste management platform, and relevant information on construction waste management facilities, including the project name, project unit, processing capacity, and floor area, is extracted. After this, the information on the location of the construction waste management facilities is converted into geographic coordinates using the forward geocoding function. Finally, the geographical coordinates are matched accurately with a map of Beijing, and a map of the distribution of construction waste management facilities in Beijing (Fig. 1) is obtained.

\subsection{Research methods}

The construction waste recycling facilities in Beijing have an agglomeration effect. If multiple recycling facilities are placed near each other, the impact will be stronger. Nearest-

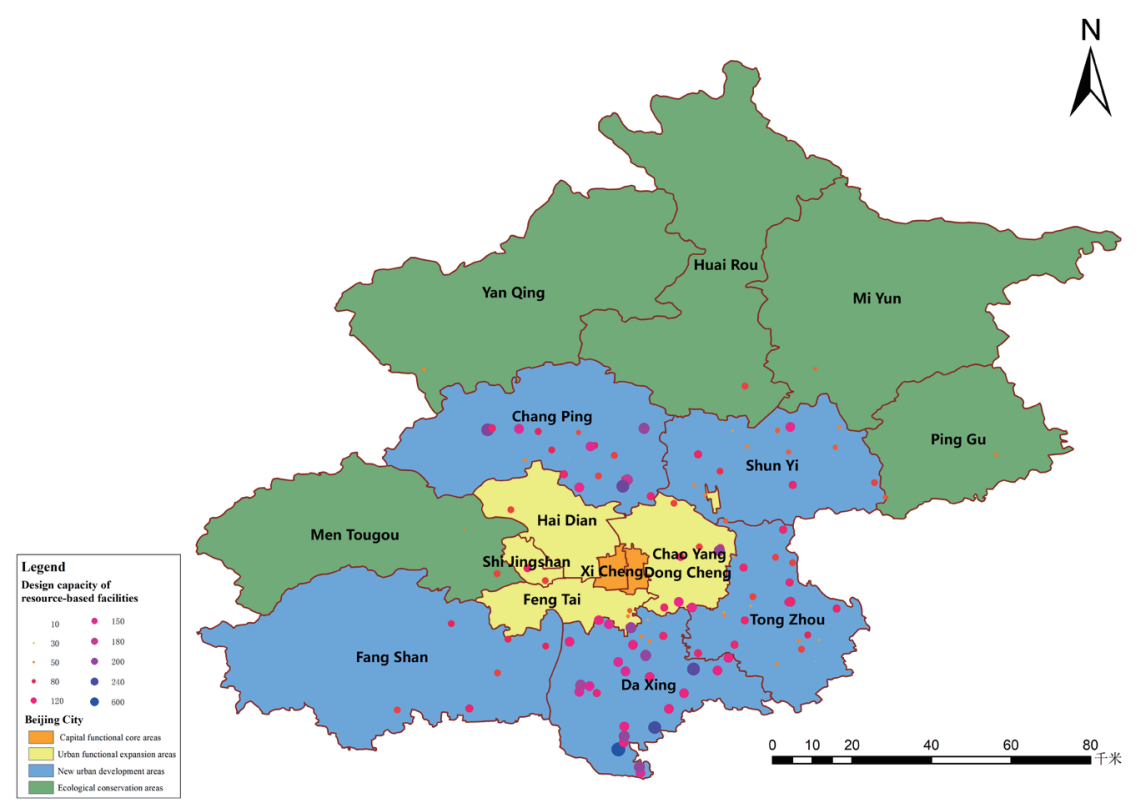

Fig. 1. (Color online) Distribution of construction waste management facilities in Beijing. 
neighbor index (NNI) and kernel density analyses are used to study the agglomeration degree of resource utilization facilities. The SDE method is one of the classic methods used to analyze the directional characteristics of spatial distributions. The size of the ellipse reflects the overall element concentration of the spatial pattern. In addition, buffer analysis is used to study the relationship between construction waste dumping points and resource facilities.

\subsubsection{NNI analysis}

The NNI quantitatively describes the distribution type and proximity of spatial point elements. ${ }^{(10)}$ By measuring the distance between the centroid of each element and the centroid of its nearest neighbor, the average value is calculated and compared with the average distance in a hypothetical random distribution. If the average distance is greater than the hypothetical distance, a cluster distribution is observed; otherwise, a decentralized distribution is observed. $Z$ is less than -0.258 and the confidence level is above $99 \% .{ }^{(10)}$ The NNI is calculated as

$$
N N I=\frac{\bar{D}_{O}}{\bar{D}_{E}}
$$

where $\bar{D}_{O}$ is the observed average distance between each point element and its nearest neighbor and $\bar{D}_{E}$ is the expected average distance between elements in a random distribution.

\subsubsection{Kernel density analysis}

Kernel density analysis is used to study the change in the spatial density of point elements, enabling the location and degree of point elements to be shown more intuitively, ${ }^{(11)}$ and is an important statistical analysis method for extracting features from a geographical spatial distribution. ${ }^{(12)}$ By taking the recycling facilities of construction waste in Beijing as the centers and the search radius as an axis with which to construct a circle, and counting the number of recycling facilities in the circle, a smooth and continuous density distribution map can be generated. Weights, which are proportional to the distance from the point event, are assigned to the surrounding area by the kernel function. ${ }^{(13)}$ If the distance attenuation is large, the area of the hot spot will be large. This is more obvious on a global scale, but it is not easy to reflect the details of the area of the hot spot. If the distance attenuation is small, the number of low- or high-value regions will be greater and the analysis will better reveal the local characteristics of the density distribution. The formula for the kernel function used in the kernel density analysis is

$$
D=\frac{3\left(1-\text { scale }^{2}\right)^{2}}{\pi r^{2}},
$$

where $r$ is the search radius and scale is the ratio of the distance from the center of the circle to the object to the search radius. 


\subsubsection{SDE analysis}

SDE analysis is an important method in spatial econometric analysis that is used to measure and analyze the characteristics of the distribution of geographical elements. ${ }^{(14)}$ The center of gravity of an SDE represents the relative position of the spatial distribution of elements, the azimuth reflects the main direction in which the distribution is trending, and the lengths of the major and minor axes are the standard distances, which represent the dispersion degrees in the main and secondary trend directions, respectively. The SDE is expressed as

$$
\begin{aligned}
& S D E_{x}=\sqrt{\frac{\sum_{i=1}^{n}\left(x_{i}-\bar{X}\right)^{2}}{n}}, \\
& S D E_{y}=\sqrt{\frac{\sum_{i=1}^{n}\left(y_{i}-\bar{Y}\right)^{2}}{n}},
\end{aligned}
$$

where $x_{i}$ and $y_{i}$ are the coordinates of elements, $\{\bar{X}, \bar{Y}\}$ represents the average center of the elements (i.e., the center of gravity), and $n$ is the total number of elements.

\subsubsection{Buffer analysis}

A buffer is defined as a buffer polygon layer with a certain width around geometric geographical elements based on the point, line, and polygon values in a database, and a composite map is created by superimposing it on a target layer. ${ }^{(15)}$ The method through which information analysis is performed is called the buffer method, and it is an important spatial operation for determining the spatial proximity and the degree of proximity among different geographical elements, and solving spatial decision making and site selection problems. ${ }^{(16)}$ The formula used in buffer analysis is

$$
P=\{x \| d(x, A) \leq r\}
$$

where $P$ is the set of buffer locations satisfying the conditions, $A$ is the research object, $d(x, A)$ is the distance from the current location to $A$, and $r$ is the radius of the neighborhood.

\section{Results and Analysis}

Through the above methods, the construction waste and its recycling facilities in Beijing are studied and analyzed. On the basis of the maximum capacity of the recycling facilities, the concentration and spatial distribution of different types of recycling facilities are discussed and 
conclusions are drawn. Finally, we study the spatial relationship between construction waste and its recycling facilities, find the deficiencies in the current distribution of recycling facilities, and propose improvements.

\subsection{Characteristics differentiating construction waste sites in Beijing}

Construction waste in Beijing is divided mainly into engineering waste, demolition waste, and decoration waste. Statistics show that at least 50 million tons of construction waste is generated in Beijing every year. ${ }^{(17)}$ On the basis of a remote sensing image of Beijing taken by Gaofen-2 in 2019, the data points for 369 construction waste storage sites in the city were vectorized by GIS spatial analysis, and a geographic database of these sites was established to allow the analysis of nuclear density and circle differentiation characteristics.

The result of the kernel density analysis is shown in Fig. 2. There are two places with a high nuclear density in the south of Beijing, at the boundary between Shi Jingshan and Feng Tai Districts and at the boundary between Tong Zhou and Chaoyang Districts. However, the nuclear density of building storage sites in the north is low and widely dispersed.

Circle analysis is helpful for revealing the differentiation characteristics of the circular layout of construction waste sites in Beijing. ${ }^{(18)}$ In this study, the former Beijing Municipal Government site was taken as the center, and a circle analysis of construction waste storage sites was conducted using radii of $5 \mathrm{~km}$ intervals. The results showed that there were 32 construction waste storage sites within a distance of less than $10 \mathrm{~km}$ from the center, accounting for $8.67 \%$ of the total, 111 sites within $10-20 \mathrm{~km}(20.08 \%$ of the total), and 130 sites within $20-30 \mathrm{~km}$, and 63,23 , and 10 such points were located within $30-40 \mathrm{~km}$ (17.07\% of the total), $40-50 \mathrm{~km}(6.23 \%)$, and beyond $50 \mathrm{~km}(2.71 \%)$, respectively. The characteristics of the circle differentiation are

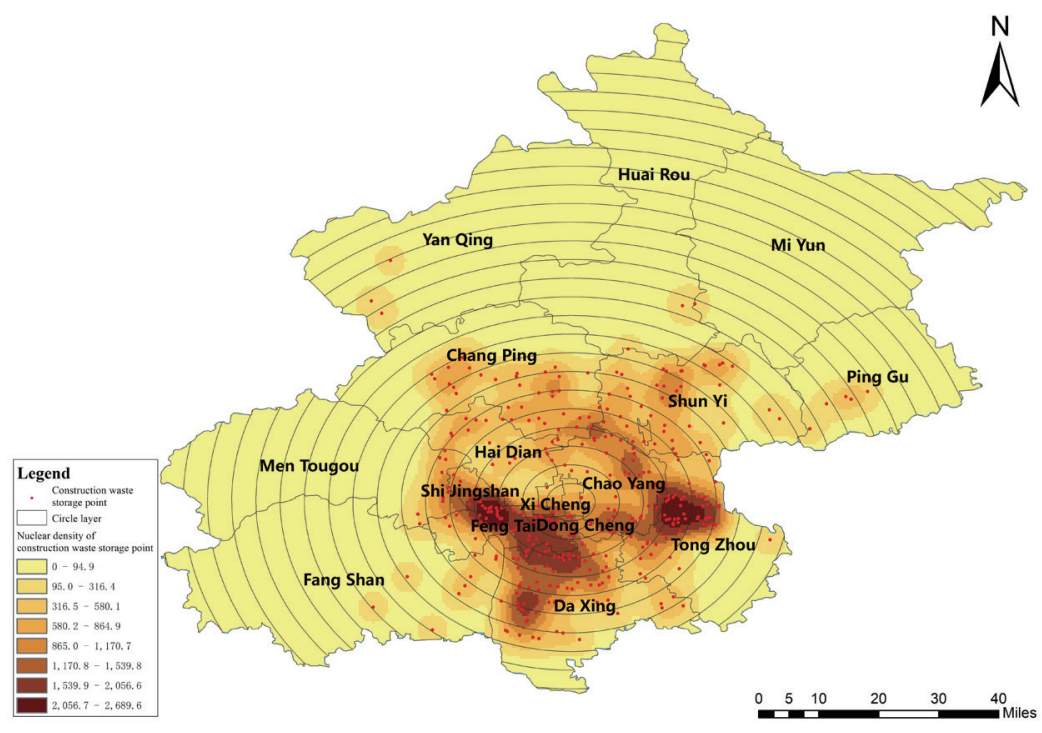

Fig. 2. (Color online) Analysis diagram showing circle differentiation and nuclear density of construction waste storage sites in Beijing. 
clear; the greatest number of construction waste storage points appears in the fourth circle layer, representing a distance of $20 \mathrm{~km}$ away from the city center and accounting for $19.51 \%$ of the total. The next largest number of points appears in the fifth circle layer, accounting for $18.97 \%$ of the total.

In addition, the numbers of construction waste storage points in the third, sixth, and seventh layers are also relatively high, exceeding $9 \%$ of the total, as shown in Fig. 3. Taking the tenth circle as the dividing line, the sum of construction waste storage points in the inner area accounts for $97.28 \%$ of the total, while the outer circle accounts for $2.71 \%$ of the total, and the number tends to remain fairly constant with further increasing distance. Therefore, the greatest amount of construction waste is produced within a ring between 10 and $30 \mathrm{~km}$ from the Beijing city center, with several storage points spread over a wide area. At the same time, the layer differentiation characteristics of the overall distribution of construction waste from the core and edge are verified. ${ }^{(19)}$

\subsection{Spatial agglomeration characteristics of construction waste recycling facilities}

The characteristics of a spatial distribution are divided into three main types: random, concentrated, and uniform. Results on the spatial distribution characteristics of construction waste recycling facilities in Beijing, as determined by NNI analysis, are shown in Table 1.

The NNI of the 115 construction waste recycling facilities in Beijing is 0.84 , which is less than 1, and the Altman $Z$ score is -3.33 , which shows that construction waste recycling facilities in Beijing show typical cluster distribution characteristics; the confidence level for this data is $99 \%$. The average observation distance of $4210 \mathrm{~m}$ indicates that the distribution density of resource facilities is high, which facilitates the treatment of construction waste in Beijing. ${ }^{(20)}$

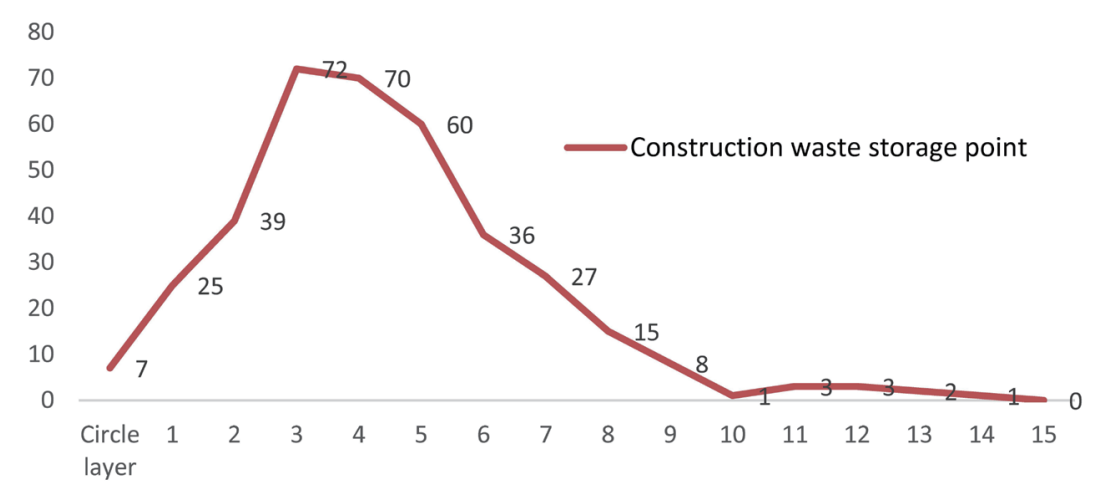

Fig. 3. (Color online) Number of construction waste storage points in different circles.

Table 1

Results of average NNI analysis.

\begin{tabular}{ccc}
\hline NNI & Altman $Z$ & $\begin{array}{c}\text { Average observation } \\
\text { distance }(\mathrm{m})\end{array}$ \\
\hline 0.84 & -3.33 & 4210 \\
\hline
\end{tabular}




\subsection{Spatial density characteristics of construction waste recycling facilities}

The results of the nuclear density analysis of construction waste recycling facilities in Beijing are shown in Fig. 4. These results reflect the local development trend. Hot spots are distributed in patches mainly concentrated in new urban development areas, with the highest density in Da Xing District followed by Chang Ping District. The large land areas of these new spaces, which are not far from the city center and are close to the urban function expansion area, allow the establishment of many fully functional resource facilities with a high processing capacity.

The treatment capacity differs among the construction waste recycling facilities. To study the spatial density characteristics of recycling facilities in further detail, the treatment plants at the recycling facilities are divided into three types, low, medium, and high, on the basis of their capacity to manage construction waste; further details are shown in Table 2.

These data show that out of the 115 construction waste recycling facilities in Beijing, most are characterized as having low and medium capacities, which are supplemented by those with a high capacity. From the perspective of the type of distribution, resource facilities with a low design capacity have a multimodal distribution, as shown in Fig. 5(a). Tong Zhou, Chang

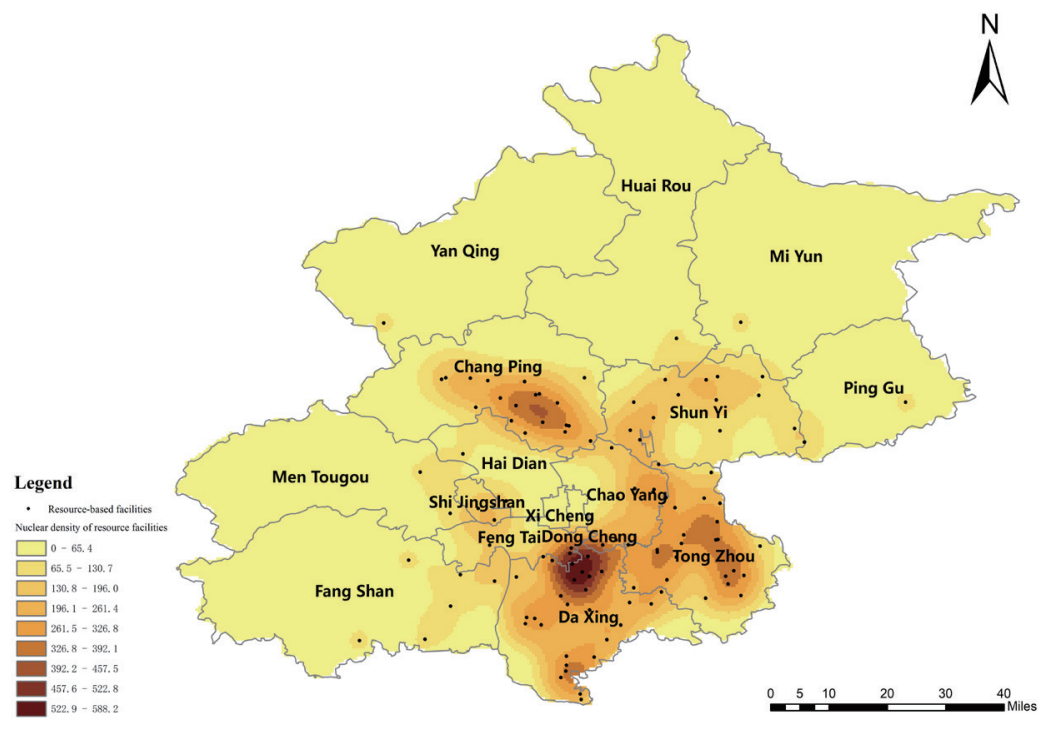

Fig. 4. (Color online) Spatial density distribution of construction waste recycling facilities in Beijing.

Table 2

Classification of design capacity of construction waste recycling facilities.

\begin{tabular}{lccl}
\hline $\begin{array}{l}\text { Capacity of resource- } \\
\text { based facility }\end{array}$ & Number & $\begin{array}{l}\text { Designed capacity } \\
(10000 \text { tons/year })\end{array}$ & \multicolumn{1}{c}{ Basis of division } \\
\hline Low & 53 & $<80$ & $\begin{array}{l}\text { Processes mainly decoration garbage, i.e., that } \\
\text { generated in the process of urban renewal. } \\
\text { Mainly for dismantling garbage: garbage that } \\
\text { cannot be disposed of in a short time can be stored } \\
\text { temporarily. } \\
\text { Medium }\end{array}$ \\
High & 13 & $80-160$ & $\begin{array}{l}\text { Mainly construction waste: time, space, and } \\
\text { specific techniques are required for processing. }\end{array}$ \\
\hline
\end{tabular}




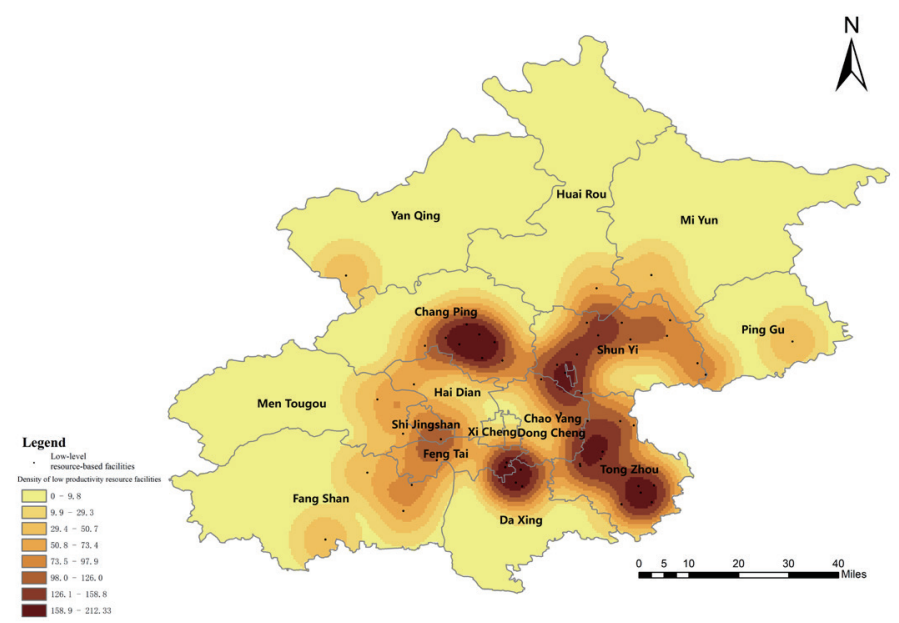

(a)

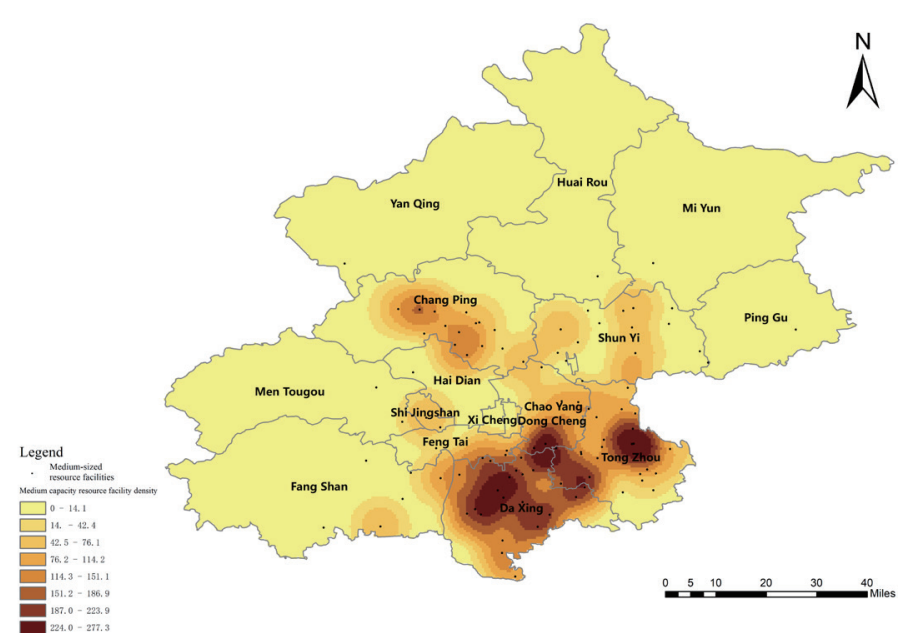

(b)

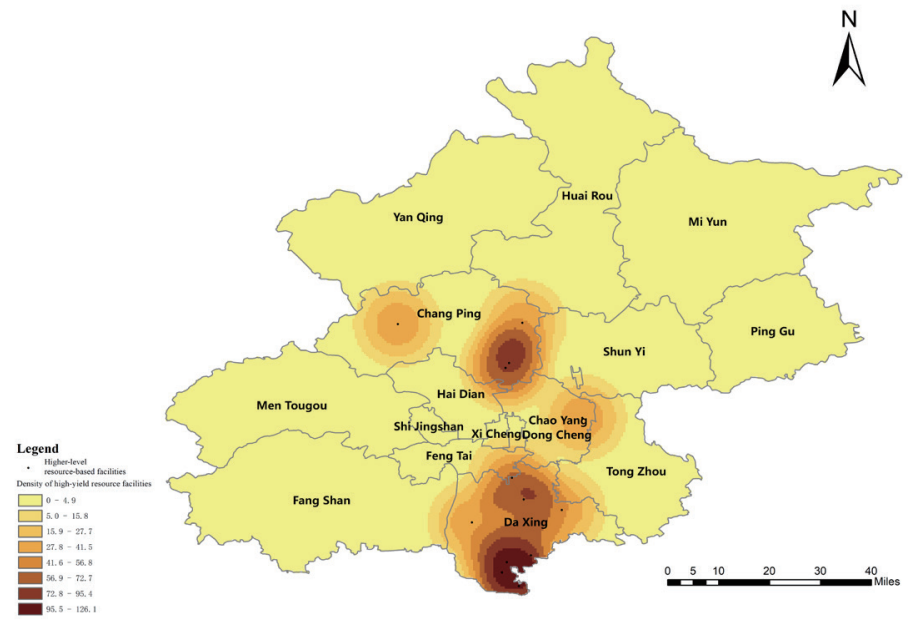

(c)

Fig. 5. (Color online) Density distributions of three different types of construction waste recycling facilities. 
Ping, and Da Xing Districts have the highest density of such facilities, followed by Shun Yi District. In 2019, the government of Beijing moved to Tong Zhou District and the State Council positioned Tong Zhou as a new city within Beijing; it is now in a period of rapid development. Chang Ping District is close to the Hai Dian Science and Technology R\&D Center, and an increasing number of enterprises are moving out from there. At the boundary between Da Xing and Feng Tai Districts, transportation is convenient, economic conditions are good, and most areas are residential; therefore, the amount of construction waste generated therein is relatively large. Shun Yi District, however, is far from the city center, has a large amount of agriculture, and has focused on the development of light industry. The amount of construction waste produced is relatively small and its distribution is scattered. Therefore, the establishment of small-scale construction waste recycling facilities around these areas can allow construction waste to be processed quickly and efficiently while reducing pollution in the city.

The resource facilities with a medium capacity are distributed in three cores concentrated mainly in Da Xing and Tong Zhou Districts, as shown in Fig. 5(b). Owing to the increase in the design capacity of resource facilities, the area they cover is relatively large and more types of construction waste are treated therein.

The resource facilities with a high capacity are distributed in a single core area, as shown in Fig. 5(c), with the highest density located in the southernmost part of Da Xing District, in a region that borders Hebei Province. This is mainly because of the design capacity, which includes several functions that allow the use of recycled construction waste. Furthermore, heavy trucks passing through $\mathrm{Da}$ Xing District allow the traffic capacity requirements to be met.

\subsection{SDE analysis of construction waste recycling facilities}

The direction distribution SDE algorithm is used to study the distribution direction characteristics of construction waste recycling facilities in Beijing. After data processing, the SDEs and their center coordinates for five types of recycling facilities with design capacities of 300000, 700000, 1 million, 1.4 million, and 2 million tons/year are obtained, ${ }^{(21)}$ as shown in Fig. 6; the corresponding data are shown in Table 3.

The average center of the ellipses of the construction waste recycling facilities is located in southeast Beijing. An analysis of the change in the shape of the SDE distribution shows that the turning angle $\theta$ of the related ellipse gradually decreases in terms of the spatial layout and that the directivity of the ellipses becomes smaller and less obvious, while the degree of data aggregation decreases continually. The standard deviation represented by the long axis increases when the design production capacity of the resource facilities increases from 300000 to 700000 tons/year, scattered in the elliptical center of each design capacity, and then decreases from 700000 to 2 million tons/year, indicating that the resource facilities are distributed along the long axis. ${ }^{(22)}$

The spatial distribution of construction waste recycling facilities considerably differs for different design capacities; those with a design capacity of 2 million tons/year have the smallest distribution and are concentrated in the southern suburbs. Those with a design capacity of 


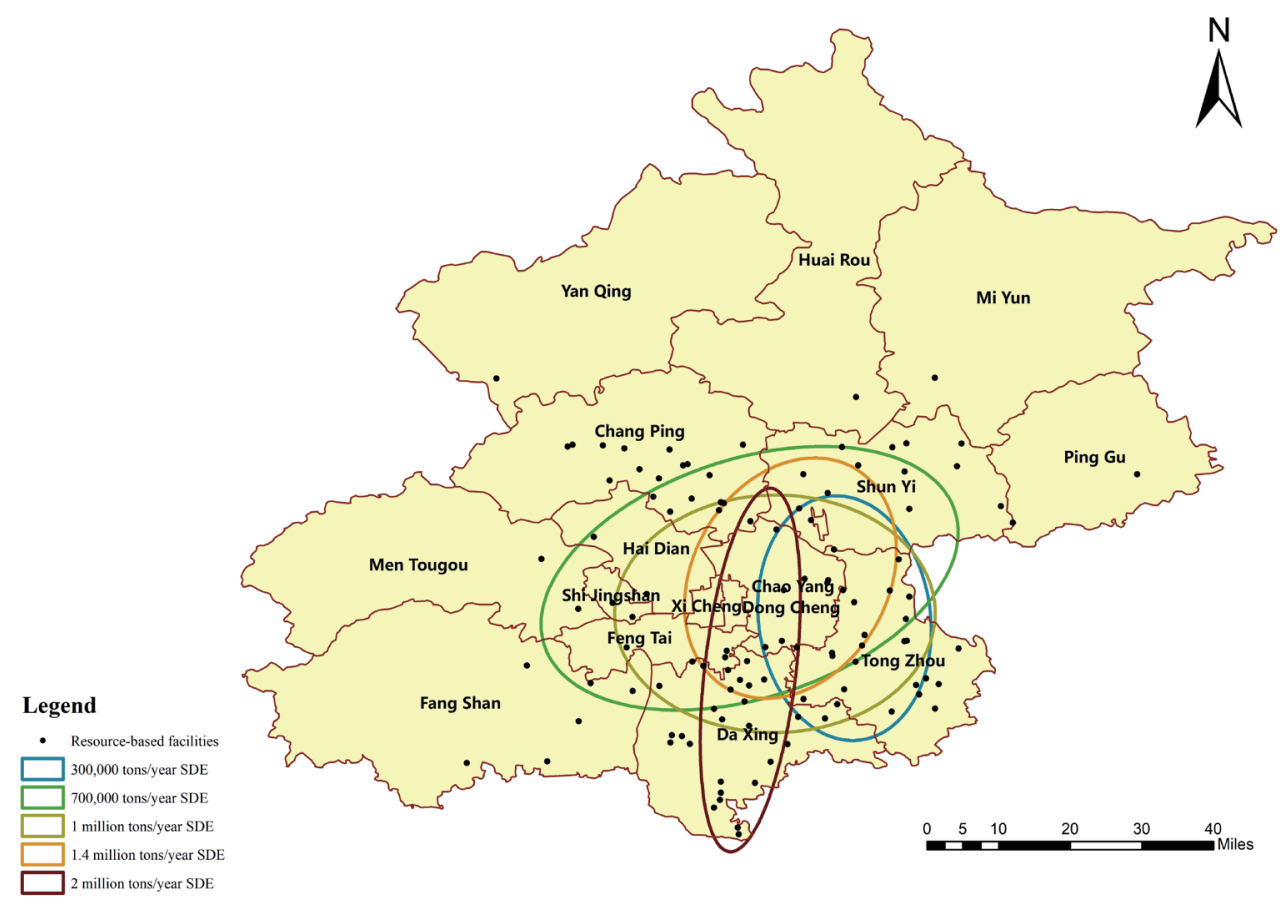

Fig. 6. (Color online) SDEs for the distribution of construction waste recycling facilities in Beijing.

Table 3

Summary of data related to SDEs for construction waste recycling facilities in Beijing.

\begin{tabular}{lllcccc}
\hline Capacity & $\begin{array}{c}\text { Gravity } \\
\text { center } X\end{array}$ & $\begin{array}{c}\text { Gravity } \\
\text { center } Y\end{array}$ & $\begin{array}{c}\text { Area } \\
\left(\mathrm{km}^{2}\right)\end{array}$ & $\begin{array}{c}\text { Long half- } \\
\text { axis }(\mathrm{km})\end{array}$ & $\begin{array}{c}\text { Short half- } \\
\text { axis }(\mathrm{km})\end{array}$ & Azimuth \\
\hline 300000 tons/year & 116.6354 & 39.8860 & 133.5 & 17.4 & 24.5 & 172.15 \\
700000 tons/year & 116.4437 & 39.9655 & 325.7 & 43.6 & 23.8 & 72.27 \\
1 million tons/year & 116.4947 & 39.8946 & 240.7 & 32.4 & 237 & 89.37 \\
1.4 million tons/year & 116.5262 & 39.9657 & 156.7 & 19.6 & 25.4 & 31.97 \\
2 million tons/year & 116.4451 & 39.7827 & 105.7 & 9.2 & 36.5 & 6.74 \\
\hline
\end{tabular}

700000 tons/year are most widely distributed in terms of direction and range; in fact, they have the largest degree of dispersion, and this is related to the distribution of construction waste storage points and the capacity of resource facilities to process construction waste.

\subsection{Connection characteristics of construction waste and its resource facilities}

Resource facilities function as treatment projects to control and treat soil pollution and improve the soil quality and environment; these facilities can also process and reuse construction waste. The service scope of Beijing construction waste resource facilities is divided according to processing capacity, transportation convenience, and traffic accessibility, as shown in Table 4. This subsection describes an analysis of the relationship between the existing construction waste recycling facilities and the spatial distribution of construction waste generated in Beijing. 
Table 4

Division of the service scope of construction waste recycling facilities in Beijing.

\begin{tabular}{lcc}
\hline $\begin{array}{l}\text { Capacity of } \\
\text { resource-based facility }\end{array}$ & $\begin{array}{c}\text { Design capacity } \\
(10000 \text { tons/year })\end{array}$ & $\begin{array}{c}\text { Scope of service } \\
(\mathrm{km})\end{array}$ \\
\hline Low & $<80$ & 2 \\
Medium & $80-160$ & 5 \\
High & $>160$ & 10 \\
\hline
\end{tabular}

Buffer analysis is carried out for the resource facilities with the three capacities, and three buffer zones are generated. The construction waste storage points are spatially connected to the buffer layer showing the resource facilities and then superimposed with the layer showing the map of Beijing to allow the analysis of the correlation among them and determine whether the existing facilities are reasonable. The results are shown in Fig. 7.

Through the attribute data table obtained after buffer analysis and space connection, the number of construction waste storage points in each resource facility buffer zone is counted and statistical tables such as Table 5 are created. The resource utilization ratio is the number of construction waste storage points contained in the layer to the total number of construction waste storage points in Beijing.

The fewest construction waste storage points are found within $2 \mathrm{~km}$ of low-capacity resource facilities owing to the large number and random distribution of these facilities, most of which are located in non-core areas of districts and counties. Most of the construction waste storage points are within $5 \mathrm{~km}$ of medium-capacity resource facilities, which have the highest proportion of resource utilization because they can process more construction waste and have a superior geographical position to low-capacity facilities. However, the service provided by construction waste recycling facilities in Beijing does not completely cover all construction waste storage sites and the number of recycling facilities in the outer suburbs is small, which leads to construction waste being piled up in the open without being processed. In addition, Hai Dian, Dong Cheng, and Xi Cheng Districts have little scope for resource-based services; therefore, the addition of resource facilities with low and medium capacities at appropriate locations can be considered. This would save transportation costs and provide a timely and localized solution to the issue of the adverse effect of construction waste on urban life.

\section{Conclusions and Suggestions}

On the basis of the empirical analysis results described above, this section gives conclusions and details of some reasonable suggestions for the layout of construction waste recycling facilities in Beijing.

\subsection{Conclusions}

The spatial distribution characteristics and factors influencing the location of construction waste recycling facilities in Beijing, against a backdrop of rapid development, were studied by spatial analysis methods such as NNI, kernel density, SDE, and buffer zone analyses. The results are as follows. 


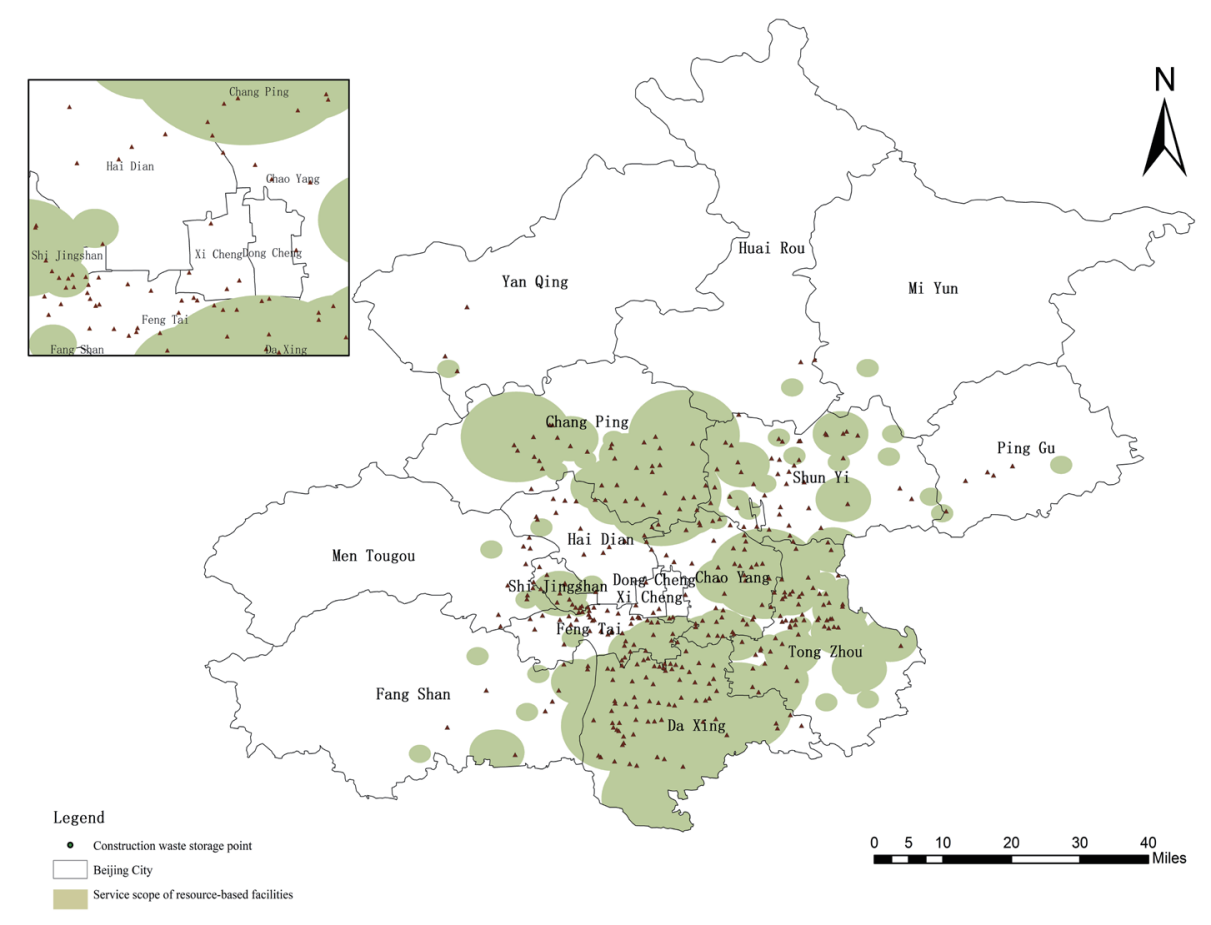

Fig. 7. (Color online) Buffer analysis of service scope of construction waste recycling facilities in Beijing.

Table 5

Statistics on buffer layer used in this study.

\begin{tabular}{lcccc}
\hline $\begin{array}{l}\text { Capacity of resource- } \\
\text { based facility }\end{array}$ & Buffer layer & $\begin{array}{c}\text { Number of } \\
\text { resource facilities }\end{array}$ & $\begin{array}{c}\text { Number of } \\
\text { construction waste } \\
\text { storage points }\end{array}$ & $\begin{array}{c}\text { Proportion of } \\
\text { resources recovered }\end{array}$ \\
\hline Low & $2 \mathrm{~km}$ & 53 & 63 & $17.1 \%$ \\
Medium & $5 \mathrm{~km}$ & 49 & 321 & $87.0 \%$ \\
High & $10 \mathrm{~km}$ & 13 & 248 & $67.2 \%$ \\
Total & Three-layer superposition & 115 & 268 & $72.6 \%$ \\
\hline
\end{tabular}

(1) The largest amount of construction waste is produced in a ring-shaped area within a radius of $10-30 \mathrm{~km}$ around the Beijing city center, where the distribution of construction waste storage sites is widespread and the proportion of such facilities in the city is high. This verifies the layer differentiation characteristics of the overall distribution of construction waste from the core and edge.

(2) There is an imbalance in the distribution of construction waste recycling facilities in Beijing, with most of them concentrated in the new urban development areas. Da Xing District has the highest density of such facilities, followed by Chang Ping District. Resource facilities with low, medium, and high processing capacities are distributed in multimodal distribution, three-core, and one-core patterns, respectively.

(3) The turning corner $\theta$ of the SDE of the spatial layout of construction waste recycling facilities gradually decreases. The standard deviation represented by the long axis increases 
when the design production capacity of the resource facilities increases from 300000 to 700000 tons/year, scattered in the elliptical center of each design capacity, and then decreases from 700000 to 2 million tons/year, indicating that the resource facilities are distributed along the long axis.

(4) There are few or no resource facilities in the outer suburbs of Beijing; therefore, the resource facilities in Hai Dian, Dong Cheng, and Xicheng Districts have limited capacities and their service scope does not completely cover all nearby construction waste storage sites, with the result that construction waste is stored in the open and not at specific sites.

At present, there are some ways in which the research in this study can be improved. In the analysis of the relationship between the construction waste recycling facilities and the spatial distribution of construction waste, only one buffer service scope scheme was used and other influencing factors were not considered in detail. Furthermore, the research on the service scope of recycling facilities should be performed in greater depth and the buffer analysis method could be improved further.

\subsection{Suggestions}

The rational layout and construction of construction waste recycling facilities are related to the prospects for development in Beijing. By relying on the support of policies, planning, information, and other industries, the integration of resource utilization and green buildings can be promoted. The examination of the layout of construction waste recycling facilities in Beijing conducted in this study presents some suggestions for the latter. Owing to the rapid pace of urban development and renewal, basic data are not updated on time, leading to some problems in the targeted consumption of resource facilities.

Beijing's urban development plan depends mostly on constructing resource facilities with a higher processing capacity to manage construction waste that cannot be reused after treatment, such as waste steel bars and broken wood. It would be appropriate to move larger resource facilities to nearby provinces and cities. Resource facilities with a medium processing capacity should cover the construction waste generated in the areas around them to the greatest extent possible so that the waste is treated locally. Finally, the many resource facilities with a low processing capacity should be updated with various advanced technologies to improve the rate at which they process construction waste with complex components.

For service scopes that are not covered by existing resource facilities, the relevant administrative departments should report accurate location details and stacking data for construction waste storage points such that the resource-based facility treatment plants can process the construction waste generated according to their own capabilities.

The resource utilization of construction waste requires further planning and design, using Internet thinking and establishing an intelligent whole-process management and control platform to reduce construction waste and resource use. 


\section{Acknowledgments}

This work was financially supported by The National Key Research and Development Program of China (2018YFC0706003) and the Pyramid Talent Training Project of Beijing University of Civil Engineering and Architecture (21082717008), and funded by the Beijing Key Laboratory of Urban Spatial Information Engineering (2020216).

\section{References}

1 H. Duan, T. R. Miller, and G. Liu: Waste Manage. 83 (2019) 1. https://doi.org/10.1016/j.wasman.2018.10.044

2 K. Povetkin and S. Isaac: J. Cleaner Prod. 266 (2020) 1. https://doi.org/10.1016/j.jclepro.2020.122024

3 Z. Bao and W. Lu: Sci. Total Environ. 724 (2020) 1. https://doi.org/10.1016/j.scitotenv.2020.138264

4 S. Weiting and W. Yunqian: Cooperative Economy Technol. 14 (2020) 179.

5 Q. Xiaofeng, L. Xiaojuan, Y. Xiaoquan, and Q. Wenwen: Reg. Res. Develop. 39 (2020) 76.

6 M. Buber, A. C. Toz, and C. Sakar: Ocean Eng. 210 (2020). https://doi.org/10.1016/j.oceaneng.2020.107576

7 J. J. Burns, R. Livingston, and R. Amin: Maternal Child Health J. 56 (2020). https://doi.org/10.1007/s10995020-02946-y

8 J. Biluca, C. R. D. Aguiar, and F. Trojan: Waste Manage. 114 (2020) 307. https://doi.org/10.1016/ j.wasman.2020.07.007

9 Z. Haokun and F. Shuangli: Environ. Hygiene Eng. 19 (2011) 63. https://doi.org/ CNKI:SUN:HJWS.0.2011-02-024

10 C. Y. Chiu, A. Prayoonwong, and Y. C. Liao: IEEE Trans. Pattern Anal. Mach. Intell. (2020) 1942. https://doi. org/10.1109/TPAMI.2019.2907086

11 D. Lili, Y. Ying, W. Lei, and C. C. Adrian: Ocean Coastal Manage. 193 (2020) 105245. https://doi.org/10.1016/ j.ocecoaman.2020.105245

12 X. Zhou, L. Xiao, and X. Lu: Growth Change 51 (2020) 852. https://doi.org/10.1111/grow.12374

13 K. G. Le, P. Liu, and L. T. Lin: Geo-spatial Inf. Sci. 23 (2020) 153. https://doi.org/10.1080/10095020.2019.1683 437

14 A. R. Cummings, N. Markandey, and H. Das: ISPRS Int. J. Geo-Inf. 8 (2019) 481. https://doi.org/10.3390/ ijgi8110481

15 W. Tasha, S. Shelly, and K. Dale: An Illustrated Dictionary of Geographic Information Systems (Esri Press, 2006). https://doi.org/10.1177/0885412208327016

16 A. A. Mohammad, T. Mohanad, and A. A. Nadhir: Sustainability 10 (2018) 2. https://doi.org/10.3390/ su10040999

17 L. Siqi: Master Thesis (Beijing Architecture University, 2020).

18 L. Jiaming, Z. Wenzhong, C. Hongxia, and Y. Jianhui: Habitat Int. 49 (2015) 33. https://doi.org/10.1016/ j.habitatint.2015.05.004

19 L. Junfu and L. Alin: Beijing Soc. Sci. 7 (2016) 72. https://doi.org/10.13262/j.bjsshkxy.bjshkx.160708

20 H. Huawei: Inf. Comput. (Theoretical Edition) 31 (2019) 141.

21 X. D. Pan, P. Li, and Z. Z. Feng: Huanjing Kexue 40 (2019) 4733 (in Chinese).

22 J. Yuan, Z. Bian, and Q. Yan: Remote Sens. 12 (2020) 945. https://doi.org/10.3390/rs12060945

\section{About the Authors}

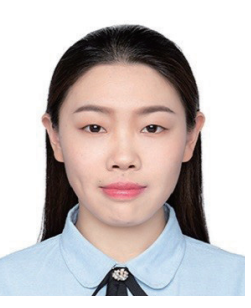

DSi-Yan Gao received her B.S. degree from Beijing University of Civil Engineering and Architecture, China, in 2019. Since then, she has been studying for a master's degree at this school. Her research interests are in smart city management applications and mobile mapping technology. (2108521519008@stu.bucea.edu.cn) 


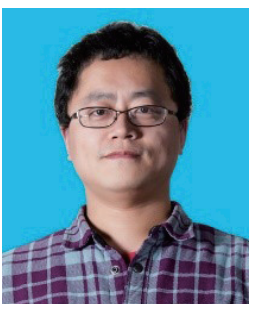

Yang Liu received his M.S. degree from Capital Normal University, China, in 2005 and his Ph.D. degree from Beijing Normal University, China, in 2008. From 2008 to 2011, he served as a lecturer at Beijing University of Civil Engineering and Architecture (BUCEA), China. He has been an assistant professor at BUCEA since 2012. His research interests are in mobile mapping technology, IOT technology, and smart city management applications. (liuyang@bucea.edu.cn)

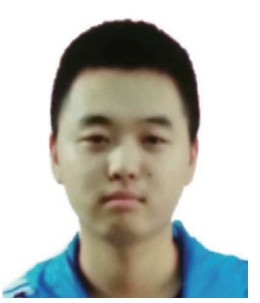

Yuan-Yang Zhou received his B.S. degree from Beijing University of Civil Engineering and Architecture, China, in 2018. Since then, he has been studying for a master's degree at this school. His research interests are in smart city management applications and IOT applications. (zhouyuanyang9@stu.bucea.edu.cn)

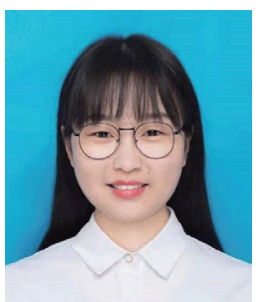

Min Zhang received her B.S. degree from Shandong University of Civil Engineering and Architecture, China, in 2018. Since then, she has been studying for a master's degree at Beijing University of Civil Engineering and Architecture, China. Her research interests are in smart city management applications and image recognition. (zhangmin@stu.bucea.edu.cn) 